\title{
Achievements of Theoretical Research on Chinese Technological Innovation
}

\author{
Hanxi Wang ${ }^{1 . *}$, Chengna $\mathrm{Wan}^{2}$, Zude Zhou ${ }^{1}$ and Shuhua $\mathrm{Hu}^{3}$ \\ ${ }^{1}$ Hubei Digital Manufacturing Key Laboratory, Wuhan University of Technology, Wuhan, China \\ ${ }^{2}$ School of Arts and Law, Wuhan University of Technology, Wuhan, China \\ ${ }^{3}$ Management College, Wuhan University of Technology, Wuhan, China \\ Corresponding author:dms2005@163.com
}

\begin{abstract}
Using the CNKI database as the sample source, we investigated the makeup of current research results on Chinese technological innovation, and found that the study of Chinese technological innovation consists of nine directions - underlying principles of innovation behavior, concepts of innovation cognition, methods of innovation, behavior of innovation subjects, structure of innovation mediators, innovation capacity, public service system of innovation, policy support system of innovation, and cultural environment of innovation, as well as several issues of innovation, including its behavior mechanism, operational mechanism, work mechanism, modes of implementation, modes of mathematical representation, modes of physical representation, modes of behavioral representation, modes of performance representation, modes of function realization, modes of structure realization, modes of workflow realization, modes of carrier realization, etc. Such a scattered makeup requires us to formulate the system of Chinese technological innovation achievements from the perspective of philosophy of science. This will be an important mission in the study of Chinese technological innovation.
\end{abstract}

\section{Introduction}

Although innovation was discussed on a large scale in China, the content of these discussions, mostly were conducted on the conception of innovation and on the purpose of innovation application. [1]

As science research, the behavioral mechanism, operational mechanism, working system and operational modes of innovation requires attention. The mathematic representative mode, physical representative modes, behavioral modes and performance representative modes of innovation need building up. The realizing modes of function, structure, procedure, embodiment of innovation need exploring [2]. From the perspective of retrieve CNKI database, the theoretical structure of China theoretical innovation research is reviewed in the paper.

\section{Research on innovation behavioral principles}

To construct innovation system on the base of innovation behavioral mechanism. This is the appropriate way for innovation to carry on from natural form to definite form. Therefore, through explorations on innovation operational rule which take as its targets the inspiring effects generated by innovation, life mechanism of innovation, working mechanism that innovation is implementing, to construct an innovation working paradigm to guide innovation development, is a key focus in China innovation theoretical research.

round the innovation mode in industrial system, Dr. Pan Xiongfeng [3], taking high-tech industry as his object, explored the life mechanism of innovation in high-tech industrial cluster, and depicted industrial cluster innovation effects by adopting innovation performance.

innovation behavioral mechanism is composed of mechanisms of impetus, matching, incentive, and operation etc. For impetus mechanism of innovation, Ji Yushan et al. [4] applied theories about social structure (i.e. evolutionary economics, technology) and organizational management in research of operation law of innovation system from intraenterprise, regional and social network to the country at the top. And they also designed a technological innovation system with dynamic mechanism in it.For the innovation match mechanism, Zhao Dumin [5] proposed a conversion and matching mechanism for technological innovation achievements through the matching between technological innovator and entrepreneur, between technological characteristics and organizational form, and between technological entrepreneurship and supporting system.

For the innovation impetus mechanism, Dr. Guo Xiaolin [6] applied the theory of behavior sciences on the sharing and diffusion mechanism of industrial common technology; adopting motivation theories proposed incentive mechanism of industrial common technology including organizational incentive, financial incentive, spiritual incentive as well as rational combination and application of each single incentive according to the change of time condition. 
Dr. Chen Fengdi [7] made a theoretical reconstruction for the operation mechanism of China technological innovation in accordance with operation mechanism of innovation system. She proposed operation mechanism of China technological innovation, composed of four parts including enterprise mechanism, government mechanism, market mechanism and social mechanism.

\section{Discussion on the concept of innovation}

Innovation is derived from discontent or dissatisfaction with present conditions, and the perception of this dissatisfaction is originated from the innovation subjects who are good at finding out problems, conflicts, and have the courage to challenge the present condition and oneself. The quality of the innovation subjects is depend on the their outlook on value conception, development conception, time conception. Therefore to expound the guiding function of innovation conception via value conception, development conception, and time conception is a fundamental issue in innovation theoretical research in China.

Dr. Lin jing [8] annotated the the possibility of science innovation through analysis of the existence significance of science innovation and demonstrated the reasons for its existence. And he also made an analysis on epistemological significance of science innovation and explained its epistemological contribution to philosophy of science and Marxist philosophy. What's more, he analyzed it on the basis of value and revealed the importance of science innovation to social progress and the all-around development of human beings.

Dr. Tian hui [9] discussed the currency significance of innovation. He proposed that innovative practice is the fundamental origin of epistemology, basic motive of epistemological development, and fundamental approach for verifying cognition; and also that cognition development is the history of innovative practice, and discussed the significance and value of innovative practice to the construction of contemporary epistemology.

Dr. Chen Li [10] discussed the time conception of innovation. He defined the scope of innovative practice from the perspective of philosophy according to the topic that innovation is core spirit of the time, and studied the structural problems of innovative practice (subject, object and intermediary), external environment problems (system, culture and resources) and mutual relationships between structure and environment. And he proposed that innovative practice is the prime impetus of human society progress, and inferred that as for society progress, practice of technological innovation is a basic impetus and practice of institutional innovation acts as an institutional impetus, while practice of intellectual innovation serves as a spiritual impetus.

\section{Research on innovation approaches}

True value of innovation research lies in making innovation a purposeful, active, automatic, orderly, normalized project activity. In order to establish a sustainable system of innovation project at human will, we need to make a deep investigation to the approaches to innovation. And only with normal innovative approaches to guide innovative project practice, can we avoid aimlessness and detours. Constructing innovative approach system is one of the key research in China innovation theory. Present researches mostly are focused on the explorations of innovation analysis approaches like its motivation analysis, risk analysis and organizational analysis.

For the motivation of innovation, Dr. Zhang Hongshi [11] constructed a motivation analysis framework of breakthrough innovation and identified a set of key motives. He found out the laws of breakthrough innovation through the interpretation of fitted curves, and set forth the breakthrough innovation mode 'Pan-Duality Organization' defined by six dimensions. And he also proved the effectiveness of 'Pan-Duality Organization' by comparison of statistic analysis and case analysis.

Around the innovation law exploration of organization structure, Dr. Liang Hongsong [12] built PAEI model in accordance with enterprise lifecycle phase judgment based on five-point scale,. And he also created an analytical model based on the organization and innovation mechanism of enterprise lifecycle.

round the exploration on innovation risk analytical approaches, Dr. Huang Yuansheng [13] constructed a selection method in technological innovation projects and an evaluation method of technological innovation capability based on the relationship between basic research and technological innovation. Meanwhile he created an identification method of technological innovation risks on the basis of the essence of technological innovation risks.

\section{Research on the behaviors of innovation agents}

Human beings are the subject of social practice, and also the agents of innovation practice. In order to inspire and achieve innovation, we need to make a research on the behavior of innovation subject inevitably. As a result, the research on the innovation behavior of innovation agents which takes traits, behavioral division, behavioral factors and leading capability of innovation subject as its parameters, is an important part of innovation theory research.

Innovative leadership is a key factor to innovation practice, so the behavior research of creative leaders is a very important aspect in the research of innovation subject. According to the motivation for innovation promotion of entrepreneurs, Dr. Guan Wei [14] described and analyzed the characters of technological innovation for entrepreneurs through four factors (variables) such as innovation motivation, innovation capability, innovation power and innovation 
decision, searched for their mutual relationship, established "an analysis model for technological innovation and enterprise scale based on control force" to make a research on dynamic relationship between technological innovation and enterprise scale in the transferring of small business to large enterprise.

Normal innovation behavior ensures stable innovation, so to construct innovation subjects' innovation behavioral norm is the ultimate goal of innovation subjects behavior. Dr. Li Zhiqiang [15] applied Principal-Agent theory in moral hazard analysis of the innovation activities. What's more, he constructed game models for describing entrepreneurs' innovation selections, and made use of institutional evolution theory analysis to propose an evolutionary model of entrepreneurs' innovation ability based on the absorption of tacit knowledge, and evolutionary logical model for institutional configuration of entrepreneurs' innovation activities.

\section{Research on the structure of innovation medium}

Innovation medium refers to the operation system established for people to implement innovation activities. The research on innovation medium is developed for the purpose of constructing a long-term, stable, creative, passionate and sustainable innovation operation project system. Generally, we need to construct the innovation operation project system in two aspects, one is project framework of innovation system including structural forms, construction paths, operation modes and tool platforms and the other, management framework of innovation system, including development strategy, behavior strategy, operational modes, and management systems. Structural framework of innovation operation project is a tool platform to achieve sustainable innovation, and also an experimental base for experimental innovation theory, applicable innovation theory and developing innovation theory, thus it is the top priority of innovation theory research.

Clear and stable innovation approach is a shortcut to implement innovation. Professor Hu Shuhua [16] created a theoretical system of product innovation management through a deep investigation of function and cost problems involved in the development and design of products under the support of Natural Science Foundation of China. His academic work, "Product Innovation and Management" published in 2000, set up a path pattern of product design and development intended for product innovation.

As the basis of standardization and engineering of innovation activity, innovation mode can provide innovation activity with behavioral paradigm. Dr. Wang Xueyuan [17] constructed a theoretical optimization framework for configuring system structure based on scientific and technological plan, in accordance with regional configuration problems of technological innovation resource. He proposed optimization rules for configuring system structure, and designed an optimization management model for configuring system structure. He also suggested a regional configuration mode of technological innovation resource and constructed a fractal evaluation model for the configuration effect of regional resources in technological innovation.

As the standard of innovation activity, innovation mode can make it follow established path, execute established regulations, use established tools and stand at established position according to project mode, in order to achieve innovation operation. However the generalization of innovation mode becomes the bottleneck for restraining its wide application. Dr. Zhao Zhenglong [18] analyzed the influence of network structure on innovation diffusion with relative advantages by constructing an innovation diffusion model based on complex social network.

Innovation mode is the high abstract for the behavior rules of innovation. It can show its value only when it is merged into specific innovation system, as a result, constructing innovation system based on innovation mode is an important topic which attracts great attention of academic circles. Dr. Liu Guoyan [19] proposed the framework, content and approaches of theoretical system for large innovative enterprises based on the two factors such as innovation and large enterprise

The building of innovation system doesn't represent launching real practice of innovation projects. The engineering of innovation not only needs innovation system, but also requires the development of innovation operation tools, the establishment of innovation operation procedures and the building of innovation operation workshop. As a result, related researches on the above three are indispensable directions of innovation research. Dr. He Shan [20] revealed the mechanism and laws of product innovation platform from the following four aspects functional combination, module distribution, level association and life cycle, and proposed three innovative behavior modes involving breakthrough, derivative and promotive product platforms. On this basis, he set forth a product innovation platform composed of management platform, information platform and technology platform.

As the basis of innovation management, innovation strategy is the strategic orientation for planning innovation activity, thus it is an important direction of innovation research. Dr. Wang Xueli [21] holds that, we can retrieve and identify innovation signals of internal and external environment from complex conditions through environmental scan and opportunity identification, then decide required extent and content of innovation from three aspects such as extant strategic evaluation, cooperative analysis of new and old strategies, as well as revolution cost of strategic innovation. Finally, we can set up new strategic location, create new business mode or construct new value network and the like, to realize effective strategic innovation.

\section{Research on innovation capacity}


Innovation capability is comprehensive subjective entity constructed by innovation subject with the support, subordination, and coordination from factors concerning innovation, and is also the premise and base to realize innovation's sustainable development, the important insurance of human civilization's sustainable progress as well. Therefore, to cultivate innovation capability of sustainable growth, is a key issue of China innovation theoretical research. From the present results of China innovation research, related China innovation capability research involves three aspects including existence forms, institutional structure and performance evaluation of innovation capability.

As for the theoretical origin of innovation capability, from the perspective of science philosophy, Dr. Lv Jianrong [22] clarified the history of innovation nation theory by historical analysis of intellectual economy, state innovation system, innovation state. He defined innovative nation by building a theoretical structure of innovative nation.

On the theory building of innovation capability, Dr. Jiang Hong [23] analyzed the application field of technological relevance and the functioning mechanism of industrial innovation and technology selection; put forward an industrial innovation model and technology selection theory model in view of technology relevance; built industry classification, industry selection model and technology selection and evaluation model based on the view of technology relevance.

On the cultivation and reproduction of innovation capabiliity, Dr. Zhang Zhongtang [24] discovered the law of enterprises' innovation system by defining the connotation of constant innovation system, put forward the functioning mechanism and structural characteristics of the leading force of enterprises' constant innovation system.

On the institutional structure of innovation capability, Dr. Chen Hong [25] found that the maintenance of stable core competitiveness depends on the knowledge innovation and knowledge management capacity of the organization; the internal knowledge innovation of the organization depends on the synergy of organization structure, organization system culture and internal knowledge network; the building of external knowledge network is a method to overcome the innovation paradox; to establish strategic knowledge network is an effective method to create stable competitive advantages.

Innovation performance is the core of innovation capability construction. So the evaluation of innovation performance is the hot issue of innovation capability theoretical research. There are four directions for the research on the evaluation of innovation capability performance, exploration on assessment mechanism, design of assessment mode, design of assessment index, design of assessment system.

On the design of performance evaluation mode of innovation capability, Dr. Sun Bing [26] built a comprehensive evaluation index system about the impetus of technological innovation of enterprises which include four levels and 30 basic indicators; he used Grid-Fuzzy Borda Number Analytical Method to give weight to each indicator in the evaluation index system, adopted principal component projection to determine the comprehensive evaluation result of impetus of technological innovation of enterprises, and hence established the comprehensive evaluation model of impetus of technological innovation of enterprises.

On the exploration on assessment of innovation capability performance, Dr. Li Qingdong [27], brought forward the evolution mechanism of industrial innovation system and synergetic evolution theory of industrial innovation system, and built a performance evaluation model and evaluation index system of industrial innovation system.

Around the design of assessment index and assessment system, on the base of scientific and technologic innovation capability structure in colleges and universities, Wang Zhangbao et al . [28] built a quantification comprehensive evaluation index system of technological innovation capability in colleges and universities according to the framework of five principles, three modules, twelve evaluation items and thirty evaluation indicators and brought forward the indicator system for the performance evaluation of scientific research of colleges and universities.

\section{Research on innovation service system}

Innovation public service system is composed of at least four essential parts, that is, innovation achievement marker service (i.e. patent service and scientific publications), innovation technology transferring service (i.e. technology exchange market), innovation information sharing service which support innovation information dissemination and technological literature sharing, innovation knowledge popularization service. As a result, how to create a innovation public service framework intending to build innovation service public platform is regarded as a direction of innovation theoretical research.

Around innovation information transmission in information sharing service, Dr. Chen Xile [29]proposed three conditions of information dissemination in network including the information finiteness of innovation subject, the desirability of knowledge innovation activity and self-organized characteristics of technological information system, and created three mechanisms of information dissemination in network, including information selection, information coordination and information feedback.

Around the construction of technological information sharing service system, Hu Limei et al. [30] raised the problems about the construction of technological information service system, including service subject, network architecture and service platform.

On the resource configuration in innovation technology transmission service and innovation knowledge popularization service, Xu Jiang et al. [31] set forth a theoretical model for the optimization configuration of regional technology innovation service resources. They argued that the input ratio of public and private innovation service resources to different innovation service fields can have significant effect on the formation of regional technology innovation service capacity. 
The ultimate goal of research on innovation public service system is the construction of innovation service platform. Zhang Qinglai et al. [32] designed an overall framework of knowledge service support system facing technological innovation, and defined the functions of each sub-system. They also proposed that on the basis of knowledge service supporting system, the knowledge service supporting hierarchy should be constructed through single-disciplinary knowledge portal, multi-disciplinary knowledge portal and even meta-knowledge portal, establishing the implement method in hierarchical construction.

\section{Research on innovation supporting policy system}

The realization of innovation needs innovation supporting policy system. The innovation supporting policy system includes innovation public policy system and government innovation management system. Which kind of innovation supporting system can stimulate innovation, and how to construct the system are the key directions of China innovation theory research.

Innovation policy means that the government, based on the acknowledgement and its demarcation of innovation value, adopts effective combination of functional macro-control methods, such as technology policy promotion, industry policy promotion, capital policy promotion and justice policy promotion. Around the synergetic promotion between technology and industry, and on the capital promotion, Wang Jingting et al. [33] analyzed the disadvantages of capital markets on each level in China from the point of supporting technological innovation, constructed multi-level capital market framework in support of technological innovation. They also made a research on the paths and policies for supporting technological innovation, involving capital markets of five levels, including main board market, growth enterprising market, agency share transfer system, property-rights exchange market and foreign capital market.

Around the government innovation management mode, Dr. Dongyou [34] discussed the relationship between technological innovation in colleges and universities and the government innovation management from the level of institutional level. He also explored government innovation management mode from two aspects: organization module and across-organization module, and designed policy adjusting tool for science innovation in colleges and universities, also developed a government macro-control evaluation index system, developed computer-aided information management system on the base of policy tool and policy match.

\section{Discussion on cultural environment of innovation}

Innovation must be supported by a wide and open cultural environment. China is an ancient nation with a history of 5000 years whose affluent cultural deposit is the base of innovation and hindrance as well.

Dr. Cui Kai [35] set up a dimensional model of enterprise innovation activities, composed of such enterprise dimensions as innovation directions, innovation behavior process, innovation risks and innovation environment for the relationship problems between innovation and culture. He explored the relationship between culture and innovation gradually with the dimensional model of enterprise innovation activities.

Dr. Cheng Liangbin [36] discussed the influence mechanism of culture on technological innovation through social capital, and illustrated the core status of cultural tradition as a basic part in Chinese technological innovation policies, and the temporal duality of cultural tradition to Chinese technological innovation policies. In the short run, cultural tradition can be regarded as a given constant, which determined our technological innovation policies; while in the long run, our technological innovation policies will have reaction to cultural tradition, making it change towards economy (non-economy but not against economy).

For the problems of relationship between social development and innovation, Dr. Liu Shiwen [37] set forth that innovation practice can be divided into three basic forms such as innovation practice in technology, institution, or knowledge, and any other non-basic forms such as innovation practice in education and art.. In his opinion, innovation practice is the natural impetus of social development.

For the purpose of constructing a humanity environment to support China innovation, Dr. Qiu Yongming [38] put forward a nation construction system which is composed of innovative nation construction soft system (innovative conception, innovative culture, innovative system, innovative intellectuals etc.)and innovative nation construction hard system (innovative system of technology, knowledge, technological and scientific state defense, region, and service system supported by science and technology).

\section{Conclusion}

The study of Chinese technological innovation consists of nine directions - underlying principles of innovation behavior, concepts of innovation cognition, methods of innovation, behavior of innovation subjects, structure of innovation mediators, innovation capacity, public service system of innovation, policy support system of innovation, and cultural environment of innovation, as well as several issues of innovation, including its behavior mechanism, operational mechanism, work mechanism, modes of implementation, modes of mathematical representation, modes of physical representation, modes of behavioral representation, modes of performance representation, modes of function realization, modes of structure realization, modes of workflow realization, modes of carrier realization, etc. 
Formulation of the Chinese technological innovation achievements framework based on the results will be a highly difficult task. Without it, scientific integration of Chinese technological innovation achievements will be difficult to accomplish, and it will be difficult for China's scientific research capacity system to serve as guidance to the investment of China's technological innovations. Therefore, the formulation of the framework from the perspective of philosophy of science will be an important mission in the study of Chinese technological innovation.

\section{References}

1. Wang Hanxi, Wan Chengna. Wuhan University of Technology Press. 406-409(2014).

2. Wang Hanxi, Zhou Zude, Hu Shuhua. Hubei People Press. 799-805(2010).

3. Pan Xiongfeng. Dalian University of Technology. 2007.

4. Ji Yushan, Zhang Zhongyu. Technoeconomics \&Management Research. 2. $23-27$ (2009).

5. Zhao Dumin. Liaoning Economy. 12. 36-37(2009).

6. Guo Xiaolin. Huazhong University of Science and Technology. 2006.

7. Chen Fengdi. Fujian Normal University. 2008.

8. Lin Jing. Jilin University. 2005.

9. Tian hui. Party School of the Central Committee of the Communist Party of China. 2008.

10. Chen Li. Party School of the Central Committee of the Communist Party of China. 2007.

11. Zhang Hongshi. Zhejiang University. 2005.

12. Liang Hongsong. Harbin Institute of Technology. 2008.

13. Huang Yuansheng. North China Electric Power University. 2005.

14. Guan Wei. Dongbei University of Finance and Economics. 2006.

15. Li Zhiqiang. Beijing Jiaotong University. 2008.

16. Hu Shuhua. Science Press. 2000.

17. Wang Xueyuan. Harbin University of Science and Technology. 2008.

18. Zhao Zhenglong. Shanghai Jiaotong University. 2008.

19. Liu Guoyan. Harbin Engineering University. 2008.

20. He Shan. Wuhan University of Technology. 2003.

21. Wang Xueli. Tianjin University. 2006.

22. Lv Jianrong. Xi'an: Xibei University. 2007.

23. Jiang Hong. Jilin University, 2008.

24. Zhang Zhongtang. Tianjin University. 2006.

25. Chen Hong. Tianjin University. 2005.

26. Sun Bing. Harbin Engineering University. 2003.

27. Li Qingdong. Jilin University. 2008.

28. Wang Zhangbao, Xu Congwei. Chinese Science \& Technology Forum. 2. 55-59(2005).

29. Chen Xile. Xiamen: Xiamen University. 2006.

30. Hu Limei, Mao Huifang, Shen Tongping. Journal of Library Science. 12. 73-75(2009).

31. Xu Jiang, Shao Yunfei. Science \& Technology Information(Academic Research). 14. 3-6(2008).

32. Zhang Qinglai, Su Yun. Library and Information. 3. 101-108(2009).

33. Wang Jingting, Wen Yue Chun. Journal of Shanghai Finance University. 1. 22-26(2009).

34. Dong You. Hebei University of Technology. 2007.

35. Cui Kai. East China Normal University. 2002.

36. Cheng Liangbin. Huazhong University of Science and Technology. 2007.

37. Liu Shiwen. Party School of the Central Committee of the Communist Party of China. 2008.

38. Qiu Yongming. East China Normal University. 2010. 